\title{
MÉTODOS PARA LA IDENTIFICACIÓN DEL ANTÍGENO DE HISTOCOMPATIBILIDAD HLA-B27: COMPARACIÓN DE CUATRO PROTOCOLOS TÉCNICOS
}

\author{
L CHILA M ${ }^{1}$, C ROMERO-SÁNCHEZ ${ }^{2}$, A GÓMEZ G ${ }^{3}$, MC CASAS $^{4}, W_{\text {BAUTISTA-MOLANO }}^{5}, \mathrm{Z}$

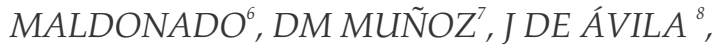 \\ I BRICEÑO ${ }^{9}$, J LONDONOO ${ }^{10}, R$ VALLE-OÑATE ${ }^{11}$.
}

${ }^{1}$ Bcl. Instructor Asistente, investigador Área Inmunogenética - Instituto UIBO, Unidad de Investigación Básica OralUniversidad El Bosque

${ }^{2} P h D$, Profesor Asociado - Universidad Militar Nueva Granada, Asesora Científica-Instituto de Referencia Andino (IRA), Coordinadora Área Inmunogenética , Instituto UIBO-Universidad El Bosque, Coordinadora Laboratorio de Inmunología Especial - Servicio de Reumatología e Inmunología, Hospital Militar Central

${ }^{3} \mathrm{PhD}$, Asesor Científico- Instituto de Referencia Andino (IRA), Profesor Titular ,Instituto de Genética Humana -

Facultad de Medicina, Pontificia Universidad Javeriana

${ }^{4} \mathrm{Bcl}$, Directora General- Instituto de Referencia Andino (IRA)

${ }^{5} \mathrm{MD}$, PhD Profesor Asociado, Facultad de Medicina - Universidad Militar Nueva Granada, Investigador-Instituto UIBO, Unidad de Investigación Básica Oral

${ }^{6} \mathrm{Bcl}$, Bacterióloga Instituto de Referencia Andino (IRA)

${ }^{7} B c l$, Bacterióloga, Servicio de Reumatología e Inmunología, Hospital Militar Central.

${ }^{8}$ Bcl, Bacterióloga, Profesor Asistente - Instituto UIBO-Universidad El Bosque

${ }^{9} \mathrm{MD}, \mathrm{PhD}$, Profesor Titular ,Instituto de Genética Humana - Facultad de Medicina, Pontificia Universidad Javeriana,

Asesor Científico- Instituto de Referencia Andino (IRA)

${ }^{10} \mathrm{MD}$, Grupo de Espondiloartropatías - Servicio de Reumatología e Inmunología, Hospital Militar Central /

Universidad de La Sabana (HMC), Profesor Titular-Universidad de la Sabana, Chía, Colombia

${ }^{11} \mathrm{MD}$, Reumatólogo, Jefe Servicio de Reumatología e Inmunología Especial, Hospital Militar Central (HMC), Profesor Asociado Facultad de Medicina - Universidad Militar Nueva Granada.

${ }^{2}$ Correspondencia: spacolombia@gmail.com

Recibido: 20 Febrero 2015 Aceptado:20 Abril 2015

\begin{abstract}
Resumen
Introducción: La asociación del HLA-B27 y las Espondiloartritis, ha hecho evidente que la tipificación del HLA-B27 sea considerada como un apoyo en el diagnóstico de estas enfermedades. Los métodos más empleados para la determinación del antígeno HLA-B27 en los laboratorios clínicos y en investigación son: la microlinfocitotoxicidad (MCTX), la citometría de flujo digital (CMFd), la citometría de flujo análoga ( $\mathrm{CMFa}$ ) y la reacción en cadena de la polimerasa con primers de secuencia específicos (PCR-SSP).

Objetivo: Comparar MCTX con la CMFd, la CMFa con la CMFd, y la técnica de CMFd frente a PCR-SSP.
\end{abstract}


Métodos: Se analizaron 4109 solicitudes de HLA-B27 en población con manifestaciones sugestivas de EAS remitidas entre 2009 y 2012 al Hospital Militar Central y al Instituto de Referencia Andino. Se evaluaron las frecuencias obtenidas por Chi cuadrado (X2); para estimar la concordancia metodológica se utilizó el Coeficiente de Correlación Intraclase (CCI). Los análisis se realizaron con el paquete estadístico SPSS V18.

Resultados: Al evaluar 467 datos por la técnica de CMFa frente a PCR-SSP, la CMFa mostró 239 resultados entre positivos y en rango indeterminado, de los cuales, luego de ser confirmados PCRSSP, solo 213 demostraron la expresión de HLA-B27 ( $<<0.05$ ). Se obtuvieron 208 resultados realizados por CMFd y PCR-SSP simultáneamente, observándose una alta correspondencia entre estas técnicas $(\mathrm{p}<0.05)$. Para evaluar la concordancia entre la MCTX y CMFd se analizaron 34 datos, revelando un $100 \%$ de correspondencia entre esta dos metodologías (CCI=1,p<0.05).

Conclusión: La citometría de flujo digital es un método rápido que presenta un desempeño altamente confiable para la identificación de HLA-B27, resultados que se recomiendan confirmar por PCR SSP.

Palabras Clave: antígeno HLA-B27, microlinfocitotoxicidad (MCTX), citometría (CMFd y CMFa), PCR-SSP

\title{
METHODS FOR IDENTIFICATION OF ANTIGEN HLA-B27 HISTOCOMPATIBILITY: FOUR TECHNICAL COMPARISONS OF PROTOCOLS
}

\begin{abstract}
Introduction: The association between HLA-B27 and spondyloarthritis has made clear the fact that identification of HLA-B27 antigen is considered as a support in the diagnosis of these diseases. The most commonly used methods for determination of the HLA-B27 antigen in clinical laboratories as well as in their research, are microlymphocytotoxicity (MCTX), digital flow cytometry (CMFd), analogous flow cytometry (CMFa) and the Single Specific Primer-Polymerase Chain Reaction (PCRSSP).

Objective: compare the CMFd against MCTX, CMFa against CMFd and CMFd against PCR-SSP.

Methods: 4109 requests for HLA-B27 were analyzed with manifestations suggestive of SpA submitted between 2009 and 2012 at Hospital Militar Central and Instituto de Referencia Andino. To analyze the frequencies Chi square (X2) was evaluated; to estimate the methodological concordance the intraclass correlation coefficient (ICC) was used. All proposed analyzes were performed with SPSS V18.

Results: 467 data obtained by CMFa versus PCR-SSP evaluated the CMFA showed 239 results between positive and indeterminate range, which, after being confirmed by molecular biology (PCRSSP), only 213 showed the expression of HLA-B27 ( $<<0.05)$. PCR-SSP and CMFd performed 208 results simultaneously, showing a high correlation between these techniques $(p<0.05)$. To evaluate the correlation between CMFd and MCTX, 34 data were analyzed, revealing a 100\% match on the positive results from these two methodologies (ICC $=1, p<0.05$ ).

Conclusion: The digital flow cytometry is a rapid method that presents a highly reliable for the initial identification of HLA-B27; results confirmed by PCR SSP recommend performance.

Keywords: HLA-B27 antigen, microlymphocytotoxicity (MCTX) cytometry (CMFd and CMFa), PCR-SSP.
\end{abstract}




\title{
MÉTODOS PARA A IDENTIFICAÇÃO DO ANTIGÉNIO DE HISTOCOMPATIBILIDADE HLA-B27: CAMPARAÇAO DE QUATRO PROTOCOLOS TÉCNICOS
}

\begin{abstract}
Resumo
Introdução: a associação do HLA-B27 $e$ as Espondilartrite, evidenciou que a tipificação do HLAB27 seja considerada como um suporte no diagnóstico dessas doenças. Os métodos mais usados para a determinação do antígeno HLA-B27 nos laboratórios clínicos e no investigação são: a microlinphocitotoxicity (MCTX), a citometria de fluxo digital (CMFd), a citometria de fluxo análoga (CMFa) e reação em cadeia de a polimerasa com primers de sequência específicos (PCR-SSP).

Objetivo: Comparar MCTX com a CMFd, a CMFa com a CMFd, e a técnica de CMFd com PCRSSP.

Métodos: 4109 solicitudes de HLA-B27 em população com manifestações sugestivas de EAS remitidas entre 2009 e 2012 ao Hospital Militar e ao Instituto de Referencia Andino, foram analisadas. Avaliaram-se as frequências obtidas por Chi quadrado (X2); para estimar a concordância metodológica foi utilizado o Coeficiente de Correlação Intraclasse (CCI). Os análises estão feitos com o paquete estadístico SPSS V18.

Resultados: A CMFa mostrou 239 resultados entre positivos e em rango indeterminado quando avaliou-se 467 dados com a técnica de CMFa com PCR-SSP. Só 213 deles demostraram a expressão de HLA-27 ( $<$ 0.05), depois de ser confirmados PCR-SSP. Foram obtidos 208 resultados por CMFd y PCR-SSP em simultâneo, com uma alta correspondência entre estas técnicas ( $p<0.05)$. Para avaliar a concordância entre MCTX y CMFd analisaram-se 34 dados, revelando um 100\% de correspondência entre as duas metodologias $(\mathrm{CCI}=, \mathrm{p}<0.05)$.

Conclusão: A citometria de fluxo é um método rápido que tem um desempeno muito confiável para a identificação de HLA-B27, resultados recomendados para confirmar por PCR SSP.

Palavras-chave: antígeno HLA-B27, microliphocitotoxicity (MCTX), citometria (CMFd y CMFa), PCR-SSP.
\end{abstract}




\section{Introducción}

La reciente utilización del antígeno HLA-B27 como ayuda diagnóstica por su inclusión dentro de los últimos criterios de clasificación para las (EAS) (1), ha enfatizado su gran importancia como biomarcador pronóstico debido a que lo presentan alrededor del $90 \%$ de pacientes espondilitis anquilosante (EA).

Es bien conocida la elevada frecuencia de este antígeno en algunos grupos étnicos, llegando hasta del 90\% de pacientes con (EA). La falta de una técnica estándar debido a los múltiples métodos empleados para su tamizaje en pacientes con sospecha de EAS, conlleva una pérdida de confiabilidad de los resultados debido a la alta variación en la sensibilidad y especificidad de los diversos protocolos técnicos utilizados, lo que dificulta el trabajo y desempeño de los laboratorios a nivel práctico y económico en busca de una medición idónea y oportuna. Como describe Bonnaud Gy "col" (2) en 1999 la tipificación de HLA-B27 contribuye al diagnóstico de la EA. La técnica clásica fue inicialmente MCTX pero esta técnica dar resultados falsos negativos ellos compararon CMF y PCR-SSP evaluando sus respectivos usos en el análisis de rutina, indicando que la tipificación de HLA-B27 por PCR SSP es una técnica confiable, pero costosa por lo tanto sugieren que puede ser utilizado como una técnica complementaria y la CMF exhibe un enfoque económico y fiable (2).

Trabajos como el de Ward y Nikaein, y de Monneret, quienes realizan una comparación entre el análisis por $\mathrm{CMF}$ frente a la MCTX concluyen que la determinación del antígeno HLA-B27 por CMF puede ser más confiable, económica y de menor tiempo de ejecución, ubicando a esta metodología como una buena alternativa aplicable a la práctica clínica y a cualquier laboratorio que esté provisto con un citómetro de flujo $(3,4)$.

Seipp y col. propusieron en el 2005 la técnica de biología molecular como estándar de oro en la determinación de HLA-B27, señalando que esta técnica es fácil de realizar y tiene una mejor sensibilidad y especificidad que los ensayos de CMF. Sin embargo, resaltan que cuando se compararon los resultados de PCR con los de la CMF, observaron una buena concordancia con excepción de cinco resultados discrepantes que se resolvieron mediante análisis de secuencia, concluyendo que aunque el protocolo de PCR para la determinación del HLA-B27 es la técnica de referencia dejan lugar a la utilización de la CMF como una técnica de buen rendimiento $(5,6)$.

Por otra parte, Nicknam y col. compararon en el 2003 la sensibilidad, la especificidad y los valores predictivos positivos y negativos de los métodos MCTX y CMF con la
PCR como método de oro en la determinación de antígeno HLA-B27. En este trabajo, utilizaron las tres técnicas mencionadas para 36 pacientes con diagnóstico confirmado de EA y 31 voluntarios sanos. Los resultados muestran que la sensibilidad, especificidad y los valores predictivos positivo y negativo de MCTX en comparación con la técnica de PCR fueron $83,3 \%, 100 \%, 100 \%$ y $88,1 \%$, respectivamente. La sensibilidad, especificidad y los valores predictivos positivo y negativo de CMF en comparación con la técnica de PCR fueron: 100\%, 94,6\%, 93,8\% y 100\%, respectivamente. Basándose en estos resultados, proponen al método de CMF como una técnica más válida para la determinación de HLA-B27 que la técnica de MCTX y de eficiencia muy cercana a la de la técnica de biología molecular (PCR-SSP) (7).

Con base en este enfoque preliminar, se compararon las metodologías utilizadas para determinar este antígeno, que incluyen técnicas celulares, serológicas y moleculares como CMF, MCTX y PCR-SSP, con el propósito de establecer el método con mejor rendimiento para la determinación del antígeno HLA-B27 en el Hospital Militar Central (HMC) y el Instituto de Referencia Andino (IRA).

\section{Materiales y Métodos}

El procesamiento de las muestras séricas se realizó en el laboratorio de inmunología del HMC y el IRA. La muestra fue sangre venosa anticoagulada con EDTA para CMF y PCR-SSP, y tubo heparinizado para MCTX, La presencia o ausencia de este antígeno se determinó en dos fases, primero por MCTX, citometría de flujo análoga/digital y luego por PCR-SSP para su confirmación. Todas las solicitudes de HLA-B27 remitidas se analizaron retrospectivamente para un total de 4109 pacientes. El proyecto contó con la aprobación de el comité de ética e investigaciones del HMC (Código 2012-085). Se realizó la evaluación de la concordancia mediante la prueba estadística de Coeficiente de Correlación Intraclase (CCI). El análisis de frecuencias se realizó con la prueba $\mathrm{X}^{2}$. Todos los análisis propuestos se realizaron con el paquete estadístico SPSS V18 para Windows.

\section{Citotoxicidad (MCTX):}

La prueba de citotoxicidad ha sido aceptada como método sencillo, sensible y reproducible para comprobar la existencia de antígeno HLA (8). El principio de la técnica de citotoxicidad es una reacción antígeno-anticuerpo, dependiente de complemento (8). La técnica original ha presentado varias modificaciones que incluyen modificación en el tiempo, en la temperatura de incubación y variaciones en la concentración de células, entre otras (9). 
Las placas de Terasaki para la tipificación de HLA vienen preparadas comercialmente con número variable de pozos (Ilustración 1) que contienen anticuerpos anti-HLA-B27 y sus diferentes subtipos en 4 a 5 pozos, además de anticuerpos que reconocen antígenos relacionados como: anti-B27+47, B2708+7, anti-B73, anti-B60+B61, antiB7, anti-B7+81+48, anti-antígenos públicos Bw4 y Bw6, así como los correspondientes controles positivos y negativos (Lymphotype HLA-B27 special Biotest ${ }^{\circledR}$ ) (10) Figura 1.

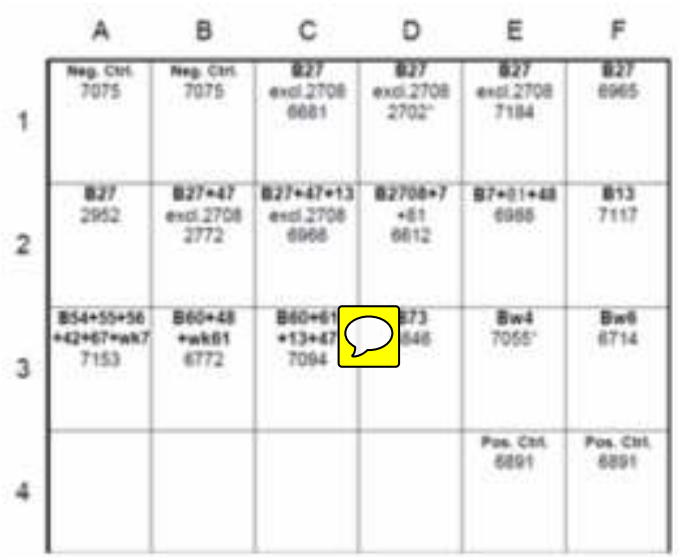

Figura 1. Placas de Terasaki de 24 pozos para la tipificación de HLAB27 (Tomado de Biotest ${ }^{\circledR}$ )

La muestra es sangre total heparinizada, se descongelaron las placas de tipificación, se separaron los mononucleares por gradiente de densidad (Ficoll hypaque ${ }^{\circledR}$ 1077), a continuación se lava la capa mononuclear 3 veces con solución salina y se resuspende en $1 \mathrm{ml}$ de solución salina. Se ajusta el número de células posterior a recuento con azul tripano; a una concentración 1,0 x 106 células/mm, a cada pozo se agrega $2 \mu \mathrm{l}$ y se incuban las placas a temperatura ambiente $(22 / 25$ c) durante 30 min, seguido se añadió $5 \mu l$ de complemento de conejo a cada uno de los pozos, se mezclan y se incuban a temperatura ambiente durante 60 minutos, después se adiciona eosina (filtrada) a cada pozo y se espera 2 min para finalmente fijar con formaldehido (37\% ph 7.0 -7.2) se cubren las placas con cubreobjetos, se deja reposar las placas a temperatura ambiente por 10-15 minutos permitiendo así el asentamiento de los linfocitos, finalmente se leyeron las placas en el microscopio de contraste de fase (10).

El método de evaluación se basa en lecturas positivas o negativas de acuerdo a la reacción o no del anticuerpo. La reacción positiva se evidencia debido al anticuerpo contra la membrana celular que se fija a la célula y por medio de la acción del complemento habrá rompimiento celular permitiendo la incorporación del colorante, esta célula se va apreciar más grande y oscura, las células vivas a parecen intactas, brillantes y refractarias. Debido a que es más fácil la interpretación, se utiliza la escala de porcentajes de células muertas con respecto al control negativo $(8,10)$.

\section{Citometría de flujo digital (CMFd)}

Un CMF FACSCanto II ${ }^{\circledR}$ fue utilizado para medir la expresión de membrana de la proteína HLA-B27. Para su detección se utilizó un anticuerpo conjugado dirigido contra la molécula de HLA-B27 acoplado a un fluorocromo. Los datos se adquirieron con el software FACSCanto ${ }^{\circledR}$, y los resultados fueron generados en gráficos y formatos de tabulaciones con base en 15000 eventos sobre población de linfocitos CD3. Los niveles son expresados como positivo o negativo $(11,12)$.(Figura 2 y 3 ).
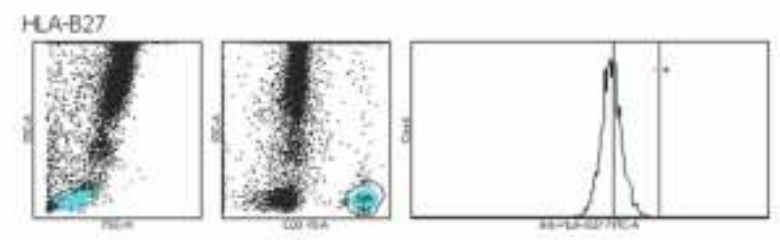

Figura 2. Citometría de flujo digital (CMFd). Lectura negativa para HLA-B27, (fotografías tomadas del archivo del Hospital Militar Central)
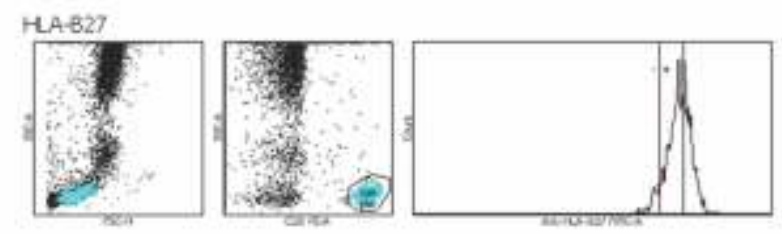

Figura 3. Citometría de flujo digital (CMFd). Lectura positiva para HLA-B27 (fotografías tomadas del archivo del Hospital Militar Central)

Las muestras de sangre venosa para la citometría de flujo digital fueron tomadas previamente en tubo con anticoagulante EDTA.

En cada tubo se dispensaron $30 \mu \mathrm{L}$ del reactivo monoclonal (Anti-HLA-B27 FITC/CD3 PE) y $50 \mu \mathrm{L}$ de la muestra de sangre con posterior mezcla en el vórtex. Los tubos se incubaron de 15 a 20 minutos a temperatura ambiente (18$25 \mathrm{C}$ ) y en un lugar protegido de la luz se añade $2 \mathrm{ml}$ del reactivo de lisis a concentración $1 \mathrm{X}$, (BD FACS lysing solution $\left.{ }^{\circledR}\right)$ y se mezcla inmediatamente en el vórtex e incuba 10 minutos a (18-25 C) en un lugar protegido de la luz. Se centrifugó 5 minutos a $300 \mathrm{~g}$ (1366 rpm) a temperatura ambiente y se eliminó el sobrenadante por aspiración dejando 50 L de sedimento para resuspender el botón celular en $3 \mathrm{~mL}$ de PBS (Facs Flow CellWASH®), con posterior mezcla e incubación 3-5 minutos y subsiguiente centrifugación 5 minutos a $300 \mathrm{~g}$ a temperatura ambiente. Se eliminó el sobrenadante por aspiración y se resuspendió 
el sedimento $(50 \mu \mathrm{L})$ en $250 \mu \mathrm{L}$ de solución PBS (Facs Flow $\left.{ }^{\circledR}\right)(11)$.

\section{Citometría de flujo análoga (CMFa):}

En la determinación del fenotipo para el HLA-B27 por CMFa se confirma la intensidad máxima de fluorescencia emitida (12). El procedimiento de identificación por CMFa se realizó a partir de sangre anticoagulada con EDTA, a cada tubo de muestra se agregan $10 \mu \mathrm{L}$ de reactivo HLA B27/B7 Coulter ${ }^{\circledR}$ y $50 \mu \mathrm{L}$ de muestra del paciente a mezclar en el vórtex. Se incubó esta mezcla 20 minutos a temperatura ambiente (18-25 C), posteriormente se agregaron $250 \mu \mathrm{L}$ de un reactivo de lisis (OptiLyse $\left.{ }^{\circledR}\right)$, se mezcló e incubó 10 minutos a temperatura ambiente. Luego se agregaron $2 \mathrm{~mL}$ de IsoFlow ${ }^{\circledR}$, y posteriormente se hizo un lavado seguido de centrifugación a $2500 \mathrm{rpm}$ por 10 min, se descartó el sobrenadante, y se resuspendió el botón con $500 \mu \mathrm{L}$ de IsoFlow $\AA$. Finalmente se procedió a la lectura en el citómetro (13) (Figura 4).
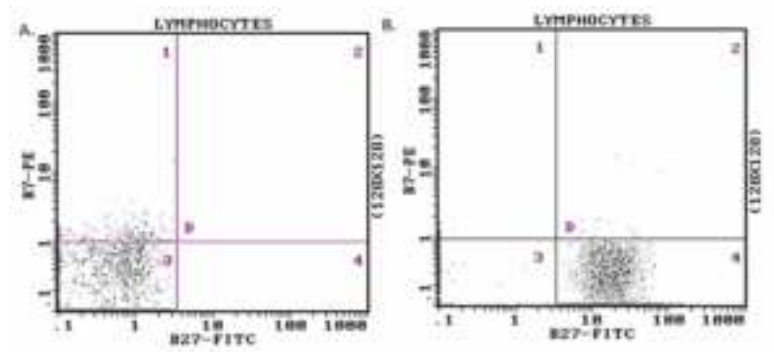

Figura 4. Citometría de flujo análoga: A) Lectura negativa para identificación de HLA-B7 y HLA-B27; B) Lectura positiva para HLA-B27 y negativa para HLA-B7 (fotografía tomadas de Iotest ${ }^{\circledR}$ HLA-B27-FITC) HLA-B7-P)

\section{PCR SSP:}

Los resultados obtenidos en las técnicas de citometría fueron confirmados por medio de la evaluación molecular del HLA-B27, tras amplificación por PCR-SSP usando el kit (Texas BioGene Inc. MorganTM HLA SSP B27 Typing). La extracción del ADN genómico se realizó a partir de sangre total anticoagulada con EDTA usando el estuche comercial o kit DNA 2000 (Corpogen DNA 2000® Ref. BM-001). La concentración final del DNA antes de la PCR-SSP fue aproximadamente $30 \mathrm{ng} / \mu \mathrm{L}$. Los alelos amplificados fueron separados en un gel de agarosa al $2 \%$, coloreado con bromuro de etidio y visualizado en transiluminador UV (14) (Figura 5).

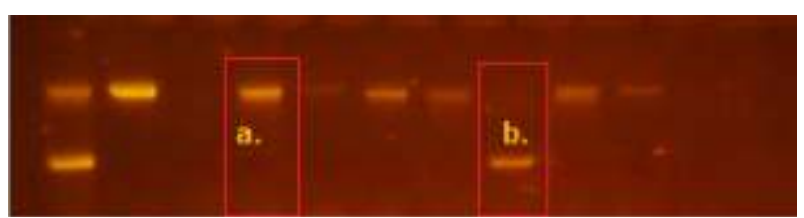

Figura 5. Fotografía de un gel de agarosa (2\%) con: a. Muestra HLA-B27 negativa y b. Muestra HLA-B27 positiva. Las muestras fueron visualizadas en transiluminador ultravioleta (UV (fotografía tomadas del archivo Hospital Militar Central)

\section{Resultados}

\section{Concordancia entre técnicas}

Para comparar el rendimiento de los métodos utilizados en este estudio fue necesario realizar un análisis de concordancia mediante la obtención del coeficiente de correlación intraclase (CCI) con un nivel de confianza del 95\%, para todos aquellos casos en donde se realizó la medición del antígeno HLA-B27 al menos por dos de las técnicas evaluadas, estableciendo como patrón de oro siempre la técnica que mostraba mayor sensibilidad, especificidad y exactitud (Tabla 1).

Tabla 1. Sensibilidad, especificidad y exactitud entre técnicas utilizadas para la detección de HLAB27

\begin{tabular}{|c|c|c|c|}
\hline IBANHCA & $\begin{array}{l}\text { SiF.NSIIHII ID.A1\} } \\
(\%)\end{array}$ & $\begin{array}{l}\text { KSIPFC IFIA IINAI } \\
(\%)\end{array}$ & $\begin{array}{l}\text { EXAC:TIIIII } \\
\mid \%)\end{array}$ \\
\hline MC I $x$ & $10 n$ & an & 100 \\
\hline C.MFa & $\$ 9$ & 200 & 44 \\
\hline C.MTL & lon & 90 & 39 \\
\hline $\mathrm{I}^{\mathrm{r}} \mathrm{CH}-\mathrm{SSI} \mathrm{I}^{3}$ & 104 & :ov & 100 \\
\hline
\end{tabular}

Para evaluar la concordancia entre la técnica de MCTX y la CMFd se analizaron 34 datos obtenidos por las dos técnicas simultáneamente revelando un $100 \%$ de correspondencia en los resultados positivos entre esta dos técnicas (CCI 1,00 $\mathrm{p}<0.05)$. Al evaluar la técnica de CMFa frente a PCR SSP, se analizaron 467 datos, una leve discrepancia en los resultados positivos y aquellos que cayeron en zona indeterminada con relación al patrón de oro observándose aun así, una correlación intraclase considerada en rangos aceptables. Esta diferencia estuvo dada por 239 resultados positivos o indeterminados obtenidos por CMFa de los cuales, luego de ser confirmados por biología molecular solo 213 demostraron la expresión de HLA-B27 (CCI 0.89 $\mathrm{p}<0.05)$.

Y finalmente para comparar CMFd y PCR-SSP simultáneamente se obtuvieron 208 resultados, de los 
cuales fueron positivos 199 y 198 respectivamente, observándose una alta consistencia entre ambas técnicas (CCI: 0,94, p<0.05). (Tabla 2)

Tabla 2. Análisis de concordancia mediante valores del Coeficiente de Correlación Intraclase (CCI).Resultados expresados únicamente sobre las pruebas positivas. ${ }^{*} p<0.05$. Resultados expresados únicamente sobre las pruebas positivas e indeterminadas $\uparrow p<0.0001$

\begin{tabular}{|c|c|c|c|}
\hline $\begin{array}{l}\text { Resultadlos } \\
\text { pesirivos por }\end{array}$ & $\begin{array}{l}\text { Resultados positivos por } \\
\text { PCR-SSP }\end{array}$ & $\mathrm{CCI}$ & $\begin{array}{l}\text { [INTERVALO DE } \\
\text { CONFLANZA } \\
95 \% \text { ! }\end{array}$ \\
\hline \multicolumn{4}{|l|}{ MCrX } \\
\hline 4 & 4 & 1 & $1-1$ \\
\hline \multicolumn{4}{|c|}{$\begin{array}{l}\text { CMFa } \\
\text { (positivese } \\
\text { indeterminados) }\end{array}$} \\
\hline 239 & 213 & $0,89^{+}$ & $0,875-0,911$ \\
\hline \multicolumn{4}{|l|}{ CMFd } \\
\hline 198 & 199 & $0,94^{*}$ & $0,928-0,958$ \\
\hline
\end{tabular}

\section{Discusión}

A lo largo del tiempo se han desarrollado diversas técnicas para la determinación del antígeno HLA-B27. Estas han mejorado su aplicabilidad clínica con base en la inclusión de esta determinación en los criterios de clasificación de las EAS y en el incremento de conocimientos sobre la importancia de este antígeno de histocompatibilidad como factor pronóstico de la enfermedad. En Colombia se han venido utilizando diversas técnicas en la evaluación de HLAB27 como MCTX, CMFa, CMFd y PCR-SSP, lo que ha implicado que los laboratorios tengan la necesidad de estandarizar estas metodologías bien como tamizaje o bien como pruebas confirmatorias cada vez que se requiera la determinación de este antígeno, para un manejo técnico en el cual los resultados obtenidos presenten el menor porcentaje de falsos positivos y/o de falsos negativos $(2,3)$. La aparición de nuevas tecnologías ha permitido la implementación de métodos con mayor sensibilidad y especificidad. En su esencia, la CMF representa un progreso técnico importante frente a la microlinfocitotoxicidad debido en gran medida a su carácter multiparamétrico en la detección de una o más características físicas y/o químicas de la superficie celular al analizar un alto número de partículas en corto tiempo. La aparición de nuevas tecnologías ha permitido la implementación de métodos con mayor sensibilidad y especificidad. En su esencia la citometría de flujo representa un proceso técnico importante debido en gran medida a su carácter multiparamétrico en la detección de una o más características físicas y/o químicas de la superficie celular analizando un alto número de partículas en corto tiempo (3). La técnica de biología molecular (PCR) es considerada como el estándar técnico de oro para la determinación de HLA-B27, tal y como se reporta en estudios como el de Bonnaud (2), Nicknam et al (7), o el de Seipp et al (6), quienes mencionan que la técnica para la determinación de HLA-B27 que tiene una mejor sensibilidad y especificidad frente a los demás ensayos es la PCR (6), los resultados del presente estudio nos demuestran una muy buena utilidad de la CMFd fundamentada en una mejor sensibilidad, objetividad, rapidez y versatilidad analítica de tamizaje frente al estudio celular. Al comparar la CMFd con la PCRSSP la concordancia obtenida muestra que los resultados adquiridos por la técnica de CMFd presentan una confiabilidad alta $(94,5 \%)$ y menos tiempo para su procesamiento.

Si bien nuestros resultados presentan a la técnica de MCTX como una buena alternativa (igual a la CMFd por su concordancia tan alta en la determinación del HLA-B27), esta técnica tiene algunas desventajas: a- Es una técnica que requiere mucho más tiempo en su ejecución, b- Tiene un porcentaje de subjetividad en su interpretación, c- Presenta reactividad cruzada con otros antígenos presentando resultados falsos negativos o positivos, y d- Está influenciada por el estado de activación de las células a analizar (7).

En cuanto a la CMFa, ésta tiene una concordancia moderada frente a la detección molecular pero presenta ciertos inconvenientes como una menor resolución y no permite modificar datos después de la adquisición. Adicionalmente, esta técnica mostró varias muestras en rango indeterminado, haciendo necesaria su confirmación y revelándose finalmente como en su mayoría negativas.

\section{Conclusiones}

La citometría de flujo digital es un método rápido que presenta mejor rendimiento frente a la citometría análoga dado que elimina los resultados en rango indeterminado confiriendo un desempeño altamente confiable para la identificación inicial del antígeno HLA-B27 y presenta una concordancia óptima con la metodología molecular. La técnica de Microlinfocitotoxicidad tiene una concordancia óptima frente a la citometría digital pero requiere de mayor tiempo de ejecución y no es posible almacenar la 
información del análisis técnico en software.

En definitiva la comparación de los resultados provenientes de la determinación de HLA-B27 de acuerdo con los diferentes parámetros permite deducir que la citometría de flujo digital es un buen método para la identificación inicial de este antígeno y la confirmación por PCR SSP proporciona resultados rápidos, óptimos y seguros con un buen rendimiento.

\section{Conflicto de intereses}

Este proyecto Fue Aprobado por comité de investigaciones del Hospital Militar Central -código 2012-084.

Informamos que durante el desarrollo de la presente investigación, ni los autores ni las instituciones que incorporamos incurrimos en ningún tipo de conflicto de intereses.

\section{Financiación}

Esta investigación no recibió financiación institucional para su realización.

\section{Referencias}

1. Bautista-Molano WA, Londoño JD, Romero C, Ávila M, Valle RR. Espondiloartritis y su asociación con el complejo mayor de histocompatibilidad. Rev Colomb Reumatol 2011;18(1):3441.

2. Bonnaud G, Aupetit C, Preux PM, Cogné M, Drouet M. Optimisation of HLA-B27 testing by association of flow cytometry and dna typing. Clin Rheumatol. 1999; 18(1):23-27.

3. Monneret G, Seffert O, Debard AL, Gutowski MC, Couprie N, Larbre JP et al. Standardization and automation of HLA-B27 typing by flow cytometry: validation and comparison with microlymphocytotoxicity. Ann Biol Clin (Paris). $2000 ; 8(4): 461-6$

4. Ward AM, Nikaein A. Comparison of monoclonal antibodies for flow cytometric analysis Of HLA-B27 Antigen. Cytometry. 1995;22(1):65-69.

5. Reynolds WM, Evans PR, Wilson PJ, Wong WM, Darke C, Smith JL. Automated routine HLA-B27 typing by flow cytometry. J Immunol Methods. 1996;197(1-2):1-5.

6. Seipp MT, Erali M, Wies RL, Wittwer C. HLA-B27 Typing: evaluation of an allele-specific PCR melting assay and two flow cytometric antigen assays. Cytometry B Clin Cytom. 2005;63(1):10-5.

7. Nicknam M.H, Jamshidi A.R, Ganjalikhani Hakemi M, Khosravi F, Amirkhani A, Narouinejad M, et al. comparison of validity of microlymphocytotoxicity and flow cytometry methods with PCR For HLA-B27 Antigen Typing. Medical Journal Of The Islamic Republic Of Iran. 2003;17(1):75-9.
8. Ávila L RC. Manual Práctico De Inmunología Clínica. Vol.1. 1aed. TM Editores,Bogotá D.C;1995.

9. Lopez-Larrea C, Gonzalez-Roces S, Peña M, Dominguez O, Coto E, Alvarez V, et al. Characterization Of B27 haplotypes by oligotyping and genomic sequencing in the mexican mestizo population with ankylosing spondylitis. Hum Immunol. 1995;43(3):174-80.

10. Biotest ${ }^{\circledR}$. Lymphotype HLA-B 27 special ref. 823110. 2006.

11. Becton Dickinson and Company. BD ${ }^{\mathrm{TM}}$ HLA-B27 KIT. 2010.

12. Dako. Dako Flow Cytometry Educational Guide`C. 2006. 2 ed. Dako Fort Collins, Colorado, USA.

13. Beckman Coulter Company. Iotest ${ }^{\circledR}$ HLA-B27-FITC/ HLAB7-P REF A07739. 2005.

14. Texas BioGene Inc. MorganTM HLA SSP B27 Typing REF $33220 / 33221$ 